\title{
Harmonic Status Index of Graphs
}

\author{
Harishchandra S. Ramane ${ }^{a}$, B. Basavanagoud ${ }^{b}$, Ashwini S. Yalnaik ${ }^{\text {* }}$ \\ Department of Mathematics, Karnatak University, Dharwad - 580003, India \\ ahsramane@yahoo.com, bb.basavanagoud@gmail.com, 'cashwiniynaik@gmail.com \\ ${ }^{*}$ Corresponding author
}

Keywords: Distance, status, diameter, harmonic status index.

\begin{abstract}
The status of a vertex $u$ is defined as the sum of the distances between $u$ and all other vertices of a graph $G$. In this paper we have defined the harmonic status index of a graph and obtained the bounds for it. Further the harmonic status indices of some graphs are obtained.
\end{abstract}

\section{Introduction}

Several distance based indices of a graph, such as Wiener index [24], distance energy [13, 18], hyper Wiener index [21], Harary index [14, 17] have been appeared in the literature. In this paper we introduce and study the new index called harmonic status index. Let $G$ be a connected graph of order $n$ and size $m$. Let $V(G)$ be the vertex set and $E(G)$ be the edge set of $G$. The edge between the vertices $u$ and $v$ is denoted by $u v$. The degree of a vertex $u$ is the number of edges incident to it and is denoted by $d(u)$. The distance between two vertices $u$ and $v$, denoted by $d(u, v)$, is the length of the shortest $u-v$ path in $G$. The maximum distance between any pair of vertices in $G$ is called the diameter of $G$ and is denoted by $\operatorname{diam}(G)$. For graph theoretic terminology, we refer the books $[1$, 2].

The status [11] of a vertex $u \in V(G)$ is defined as the sum of its distance from every other vertex in $V(G)$ and is denoted by $\sigma(u)$. That is,

$$
\sigma(u)=\sum_{v \in V(G)} d(u, v)
$$

The Wiener index [24] $W(G)$ of a connected graph $G$ is defined as the sum of the distances between all pairs of vertices of $G$. That is,

$$
W(G)=\sum_{\{u, v\} \subseteq V(G)} d(u, v)=\frac{1}{2} \sum_{u \in V(G)} \sigma(u) .
$$

For more about the Wiener index one can refer [4, 7, 10, 19, 20, 23].

The harmonic index of a graph $G$ is defined as [8]

$$
H(G)=\sum_{u v \in E(G))} \frac{2}{d(u)+d(v)} .
$$

Recent results on the harmonic index can be found in $[3,5,12,15,16,22,25,26]$. Inspired by this definition, we define here harmonic status index of a connected graph $G$ as

$$
H S(G)=\sum_{u v \in E(G))} \frac{2}{\sigma(u)+\sigma(v)},
$$

and obtain the bounds for the harmonic status index. Also we obtain the harmonic status index of some graphs. Further the correlation between the boiling point of paraffins and harmonic status index of the corresponding molecular graph is studied. 
For a graph given in Fig. $1, \sigma\left(u_{1}\right)=5, \sigma\left(u_{2}\right)=3, \sigma\left(u_{3}\right)=4, \sigma\left(u_{4}\right)=4$. And

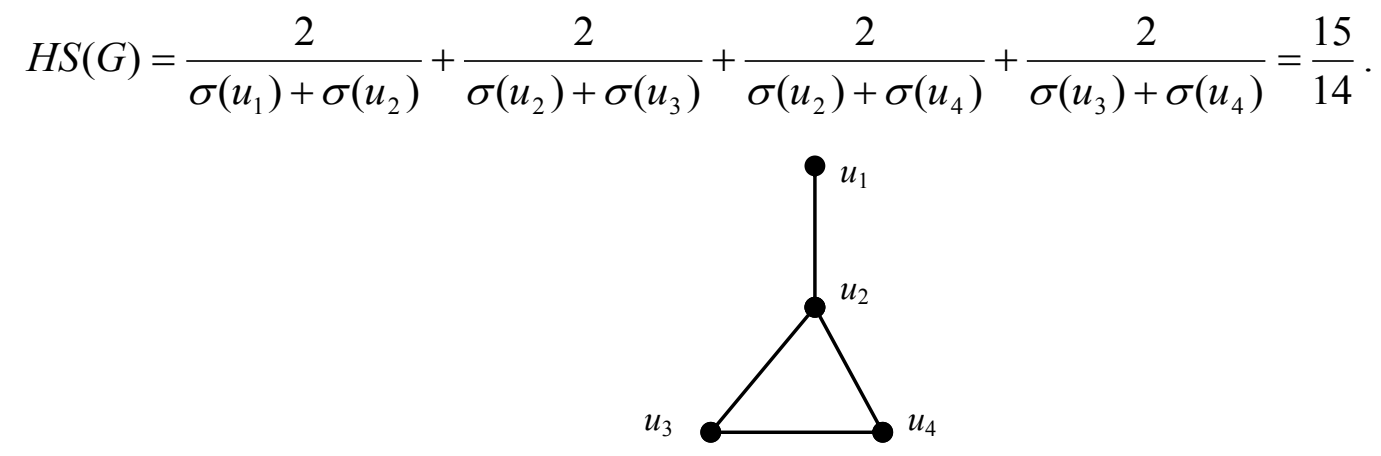

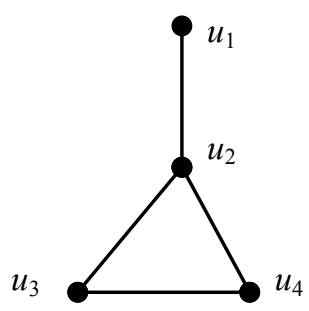

Fig. 1. Graph

\section{Bounds for the harmonic status index}

In this section we obtain the bounds for the harmonic status index of graphs and characterize for the equality of these bonds.

Theorem 1. Let $G$ be a connected graph with $n$ vertices and let $\operatorname{diam}(G)=D$. Then

$$
\sum_{u v \in E(G)} \frac{2}{2 D(n-1)-(D-1)[d(u)+d(v)]} \leq H S(G) \leq \sum_{u v \in E(G)} \frac{2}{4 n-4-[d(u)+d(v)]} .
$$

Equality on both sides holds if and only if $\operatorname{diam}(G) \leq 2$.

Proof. Lower bound: For any vertex $u$ of $G$, there are $d(u)$ vertices which are at distance 1 from $u$ and the remaining $(n-1-d(u))$ vertices are at distance at most $D$. Therefore

$$
\sigma(u) \leq d(u)+D(n-1-d(u))=D(n-1)-(D-1) d(u) .
$$

Therefore $\sigma(u)+\sigma(v) \leq 2 D(n-1)-(D-1)[d(u)+d(v)]$. Therefore

$$
H S(G)=\sum_{u v \in E(G)} \frac{2}{\sigma(u)+\sigma(v)} \geq \sum_{u v \in E(G)} \frac{2}{2 D(n-1)-(D-1)[d(u)+d(v)]} .
$$

Upper bound: For any vertex $u$ of $G$, there are $d(u)$ vertices which are at distance 1 from $u$ and the remaining $(n-1-d(u))$ vertices are at distance at least 2 . Therefore

$$
\sigma(u) \geq d(u)+2(n-1-d(u))=2 n-2-d(u) .
$$

Therefore $\sigma(u)+\sigma(v) \geq 4 n-4-[d(u)+d(v)]$. Therefore

$$
H S(G)=\sum_{u v \in E(G)} \frac{2}{\sigma(u)+\sigma(v)} \leq \sum_{u v \in E(G)} \frac{2}{4 n-4-[d(u)+d(v)]} .
$$

For equality: If the diameter $D$ is 1 or 2 then the equality holds.

Conversely, let

$$
H S(G)=\sum_{u v \in E(G)} \frac{2}{4 n-4-[d(u)+d(v)]} .
$$

Suppose, $D \geq 3$, then there exists at least one pair of vertices $u$ and $v$ such that $d(u, v) \geq 3$. Therefore $\sigma(u) \geq d(u)+3+2(n-2-d(u))=2 n-1-d(u)$. Therefore

$$
H S(G) \leq \sum_{u v \in E(G)} \frac{2}{4 n-2-[d(u)+d(v)]}<\sum_{u v \in E(G)} \frac{2}{4 n-4-[d(u)+d(v)]},
$$

a contradiction. Hence $\operatorname{diam}(G) \leq 2$. 
Corollary 1.1. Let $G$ be a connected graph with $n$ vertices, $m$ edges and $\operatorname{diam}(G)=D$. Let $\delta$ and $\Delta$ be the minimum and maximum degree of the vertices of $G$ respectively. Then

$$
\frac{m}{D(n-1)-(D-1) \delta} \leq H S(G) \leq \frac{m}{2 n-2-\Delta} .
$$

Proof. For any vertex $u$ of $G, d(u) \geq \delta$ and $d(u) \leq \Delta$. Therefore substituting $d(u)+d(v) \geq 2 \delta$ on left side and $d(u)+d(v) \leq 2 \Delta$ on the right side of Eq. (1) we get the result.

Corollary 1.2. Let $G$ be a connected regular graph of degree $r$ with $n$ vertices, $m$ edges and $\operatorname{diam}(G)$ $=D$. Then

$$
\frac{m}{D(n-1)-(D-1) r} \leq H S(G) \leq \frac{m}{2 n-2-r} .
$$

Equality holds if and only if $\operatorname{diam}(G) \leq 2$.

Proof. For any vertex $u$ of $G, d(u)=r$. Therefore the result follows from the Theorem 1 .

\section{Harmonic status index of some standard graphs}

Proposition 2. For a complete graph $K_{n}$ on $n$ vertices, $H S\left(K_{n}\right)=n / 2$.

Proof. For any vertex $u$ of $K_{n}, \sigma(u)=n-1$. Therefore by the definition of harmonic status index, $H S\left(K_{n}\right)=n / 2$.

Proposition 3. For a complete bipartite graph $K_{p, q}$,

$$
H S\left(K_{p, q}\right)=\frac{2 p q}{3(p+q)-4} .
$$

Proof. The vertex set $V\left(K_{p, q}\right)$ can be partitioned into two independent sets $V_{1}$ and $V_{2}$ such that for every edge $u v$ of $K_{p, q}$, the vertex $u \in V_{1}$ and $v \in V_{2}$. Therefore $d(u)=q$ and $d(v)=p$. The graph $K_{p, q}$ has $n=p+q$ vertices and $m=p q$ edges. Also $\operatorname{diam}\left(K_{p, q}\right) \leq 2$. Therefore by the equality part of Theorem 1,

$$
H S\left(K_{p, q}\right)=\sum_{u v \in E\left(K_{p, q}\right)} \frac{2}{4(p+q)-4-[q+p]}=\frac{2 p q}{3(p+q)-4}
$$

Proposition 4. For a path $P_{n}$ on $n$ vertices,

$$
H S\left(P_{n}\right)=\sum_{i=1}^{n-1} \frac{2}{(n-i)^{2}+i^{2}}
$$

Proof. Let $v_{1}, v_{2}, \ldots, v_{n}$ be the vertices of $P_{n}$, where $v_{i}$ is adjacent to $v_{i+1}, i=1,2, \ldots, n-1$. Therefore for $i=1,2, \ldots, n-1$,

$$
\sigma\left(v_{i}\right)=(i-1)+(i-2)+\cdots+1+1+2+\cdots(n-i)=\frac{n^{2}+n}{2}+i(i-n-1) .
$$

Therefore

$$
H S\left(P_{n}\right)=\sum_{i=1}^{n-1} \frac{2}{\sigma\left(v_{i}\right)+\sigma\left(v_{i+1}\right)}=\sum_{i=1}^{n-1} \frac{2}{(n-i)^{2}+i^{2}} .
$$


Proposition 5. For a cycle $C_{n}$ on $n \geq 3$ vertices,

$$
H S\left(C_{n}\right)= \begin{cases}\frac{4}{n} & \text { if } n \text { is even } \\ \frac{4 n}{n^{2}-1} & \text { if } n \text { is odd. }\end{cases}
$$

Proof. If $n$ is even number then for any vertex $u$ of $C_{n}$,

$$
\sigma(u)=2\left[1+2+\cdots+\frac{n-1}{2}\right]+\frac{n}{2}=\frac{n^{2}}{4} .
$$

Therefore

$$
H S\left(C_{n}\right)=\sum_{u v \in E\left(C_{n}\right)} \frac{2}{\sigma(u)+\sigma(v)}=\frac{4}{n} .
$$

If $n$ is odd number then for any vertex $u$ of $C_{n}$,

$$
\sigma(u)=2\left[1+2+\cdots+\frac{n-1}{2}\right]=\frac{\left(n^{2}-1\right)}{4} .
$$

Therefore

$$
H S\left(C_{n}\right)=\sum_{u v \in\left(C_{n}\right)} \frac{2}{\sigma(u)+\sigma(v)}=\frac{4 n}{n^{2}-1} .
$$

A wheel $W_{n+1}$ is a graph obtained from the cycle $C_{n}, n \geq 3$ by adding a new vertex and making it adjacent to all the vertices of $C_{n}$. The degree of a central vertex of $W_{n+1}$ is $n$ and the degree of all other vertices is 3 .

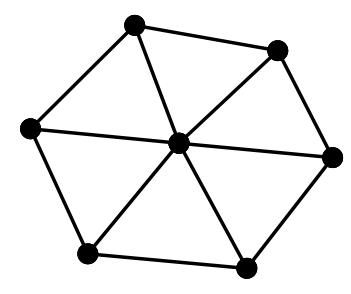

Fig. 2. Wheel $W_{7}$

Proposition 6. For a wheel $W_{n+1}, n \geq 3$,

$$
H S\left(W_{n+1}\right)=\frac{n(7 n-9)}{3(n-1)(2 n-3)} .
$$

Proof. Partition the edge set $E\left(W_{n+1}\right)$ into two sets $E_{1}$ and $E_{2}$, where $E_{1}=\{u v \mid d(u)=n$ and $d(v)=3\}$ and $E_{2}=\{u v \mid d(u)=3$ and $d(v)=3\}$. It is easy to check that $\left|E_{1}\right|=n$ and $\left|E_{2}\right|=n$. Also $\operatorname{diam}\left(W_{n+1}\right)=$ 2.

Therefore by the equality part of Theorem 1,

$$
\begin{aligned}
& H S\left(W_{n+1}\right)=\sum_{u v \in E_{1}} \frac{2}{4(n+1)-4-[d(u)+d(v)]}+\sum_{u v \in E_{2}} \frac{2}{4(n+1)-4-[d(u)+d(v)]} \\
= & \frac{n(7 n-9)}{3(n-1)(2 n-3)} .
\end{aligned}
$$


A friendship graph (or Dutch windmill graph) $F_{n}, n \geq 2$, is a graph that can be constructed by coalescence $n$ copies of the cycle $C_{3}$ of length 3 with a common vertex. It has $2 n+1$ vertices and $3 n$ edges. The degree of a coalescence vertex of $F_{n}$ is $2 n$ and the degree of all other vertices is 2 .

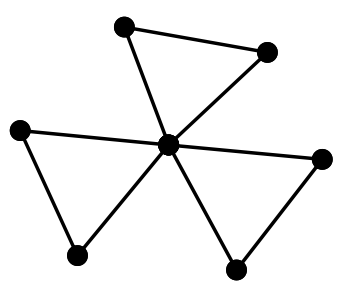

Fig. 3. Friendship graph $F_{3}$

Proposition 8. For a friendship graph $F_{n}, n \geq 2$,

$$
H S\left(F_{n}\right)=\frac{n(11 n-5)}{(3 n-1)(4 n-2)} .
$$

Proof. Partition the edge set $E\left(F_{n}\right)$ into two sets $E_{1}$ and $E_{2}$, where $E_{1}=\{u v \mid d(u)=2 n$ and $d(v)=2\}$ and $E_{2}=\{u v \mid d(u)=2$ and $d(v)=2\}$. It is easy to check that $\left|E_{1}\right|=2 n$ and $\left|E_{2}\right|=n$. Also $\operatorname{diam}\left(F_{n}\right)=$ 2.

Therefore, by the equality part of Theorem 1,

$$
\begin{aligned}
& H S\left(F_{n}\right)=\sum_{u v \in E_{1}} \frac{2}{4(2 n+1)-4-[d(u)+d(v)]}+\sum_{u v \in E_{2}} \frac{2}{4(2 n+1)-4-[d(u)+d(v)]} \\
= & \frac{n(11 n-5)}{(3 n-1)(4 n-2)} .
\end{aligned}
$$

\section{Harmonic status index of some graphs obtained from the complete graph}

In this section we obtain the harmonic status index of the graphs, which were defined in [9].

Proposition 9. Let $e_{i}, i=1,2, \ldots, k, 1 \leq k \leq n-2$, be the distinct edges of a complete graph $K_{n}$, $n \geq 3$, all being incident to a single vertex. The graph $K a_{n}(k)$ is obtained by deleting $e_{i}, i=1,2, \ldots$, $k$ from $K_{n}$. Then

$$
H S\left(K a_{n}(k)\right)=\frac{2(n-k-1)}{2 n-2+k}+\frac{k(k-1)}{2 n}+\frac{2(n-k-1) k}{2 n-1}+\frac{(n-k-1)(n-k-2)}{2 n-2} .
$$

Proof. The edge set $E\left(K a_{n}(k)\right)$ can be partitioned into four sets $E_{1}, E_{2}, E_{3}$ and $E_{4}$, where $E_{1}=\{u v \mid$ $d(u)=n-1-k$ and $d(v)=n-1\}, E_{2}=\{u v \mid d(u)=n-2$ and $d(v)=n-2\}, E_{3}=\{u v \mid d(u)=n-2$ and $d(v)=n-1\}$ and $E_{4}=\{u v \mid d(u)=n-1$ and $d(v)=n-1\}$. It is easy to check that $\left|E_{1}\right|=n-k-$ $1,\left|E_{2}\right|=k(k-1) / 2,\left|E_{3}\right|=(n-k-1) k$ and $\left|E_{4}\right|=(n-k-1)(n-k-2) / 2$. Also $\operatorname{diam}\left(K a_{n}(k)\right)=2$.

Therefore, by the equality part of Theorem 1 ,

$$
\begin{aligned}
& H S\left(K a_{n}(k)\right)=\sum_{u v \in E_{1}} \frac{2}{4 n-4-[d(u)+d(v)]}+\sum_{u v \in E_{2}} \frac{2}{4 n-4-[d(u)+d(v)]} \\
& +\sum_{u v \in E_{3}} \frac{2}{4 n-4-[d(u)+d(v)]}+\sum_{u v \in E_{4}} \frac{2}{4 n-4-[d(u)+d(v)]}
\end{aligned}
$$




$$
\begin{aligned}
& =\frac{2(n-k-1)}{2 n-2+k}+\frac{2(k(k-1) / 2)}{2 n}+\frac{2(n-k-1) k}{2 n-1}+\frac{2((n-k-1)(n-k-2) / 2)}{2 n-2} \\
& =\frac{2(n-k-1)}{2 n-2+k}+\frac{k(k-1)}{2 n}+\frac{2(n-k-1) k}{2 n-1}+\frac{(n-k-1)(n-k-2)}{2 n-2} .
\end{aligned}
$$

Proposition 10. Let $f_{i}, i=1,2, \ldots, k, 1 \leq k \leq\lfloor n / 2\rfloor$ be independent edges of the complete graph $K_{n}, n \geq 3$. The graph $K b_{n}(k)$ is obtained by deleting $f_{i}, i=1,2, \ldots, k$ from $K_{n}$. Then

$$
H S\left(K b_{n}(k)\right)=\frac{4 k(n-2 k)}{2 n-1}+\frac{(n-2 k)(n-2 k-1)}{2 n-2}+\frac{2 k(k-1)}{n} .
$$

Proof. The edge set $E\left(K b_{n}(k)\right)$ can be partitioned into three sets $E_{1}, E_{2}$, and $E_{3}$, where $E_{1}=\{u v \mid d(u)$ $=n-2$ and $d(v)=n-1\}, E_{2}=\{u v \mid d(u)=n-1$ and $d(v)=n-1\}$, and $E_{3}=\{u v \mid d(u)=n-2$ and $d(v)=n-2\}$. It is easy to check that $\left|E_{1}\right|=2 k(n-2 k),\left|E_{2}\right|=(n-2 k)(n-2 k-1) / 2$, and $\left|E_{3}\right|=(2 k(2 k$ $-1) / 2)-k$. Also $\operatorname{diam}\left(K b_{n}(k)\right)=2$.

Therefore, by the equality part of Theorem 1 ,

$$
\begin{aligned}
H S\left(K b_{n}(k)\right) & =\sum_{u v \in E_{1}} \frac{2}{4 n-4-[d(u)+d(v)]}+\sum_{u v \in E_{2}} \frac{2}{4 n-4-[d(u)+d(v)]}+\sum_{u v \in E_{3}} \frac{2}{4 n-4-[d(u)+d(v)]} \\
& =\frac{2(2 k)(n-2 k)}{2 n-1}+\frac{2((n-2 k)(n-2 k-1) / 2)}{2 n-2}+\frac{2((2 k(2 k-1) / 2)-k)}{2 n} \\
& =\frac{4 k(n-2 k)}{2 n-1}+\frac{(n-2 k)(n-2 k-1)}{2 n-2}+\frac{2 k(k-1)}{n} .
\end{aligned}
$$

Proposition 11. Let $V_{k}$ be a $k$-element subset of the vertex set of the complete graph $K_{n}, 2 \leq k \leq n-$ $1, n \geq 3$. The graph $K c_{n}(k)$ is obtained by deleting from $K_{n}$ all the edges connecting pairs of vertices from $V_{k}$. Then

$$
H S\left(K c_{n}(k)\right)=\frac{2 k(n-k)}{2 n+k-3}+\frac{(n-k)(n-k-1)}{2 n-2} .
$$

Proof. The edge set $E\left(K c_{n}(k)\right)$ can be partitioned into two sets $E_{1}$, and $E_{2}$, where $E_{1}=\{u v \mid d(u)=n$ $-k$ and $d(v)=n-1\}$ and $E_{2}=\{u v \mid d(u)=n-1$ and $d(v)=n-1\}$. It is easy to check that $\left|E_{1}\right|=(n-$ $k) k$ and $\left|E_{2}\right|=(n-k)(n-k-1) / 2$. Also $\operatorname{diam}\left(K c_{n}(k)\right)=2$.

Therefore, by the equality part of Theorem 1,

$$
\begin{aligned}
H S\left(K c_{n}(k)\right) & =\sum_{u v \in E_{1}} \frac{2}{4 n-4-[d(u)+d(v)]}+\sum_{u v \in E_{2}} \frac{2}{4 n-4-[d(u)+d(v)]} \\
& =\frac{2(n-k) k}{2 n+k-3}+\frac{2((n-k)(n-k-1) / 2)}{2 n-2} \\
& =\frac{2 k(n-k)}{2 n+k-3}+\frac{(n-k)(n-k-1)}{2 n-2} .
\end{aligned}
$$

Proposition 12. Let $3 \leq k \leq n, n \geq 5$. The graph $K d_{n}(k)$ is obtained by deleting from $K_{n}$, the edges belonging to a $k$-membered cycle. Then

$$
H S\left(K d_{n}(k)\right)=\frac{k(k-3)}{2 n+2}+\frac{k(n-k)}{n}+\frac{(n-k)(n-k-1)}{2 n-2} .
$$


Proof. The edge set $E\left(K d_{n}(k)\right)$ can be partitioned into three sets $E_{1}, E_{2}$ and $E_{3}$, where $E_{1}=\{u v \mid d(u)$ $=n-3$ and $d(v)=n-3\}, E_{2}=\{u v \mid d(u)=n-3$ and $d(v)=n-1\}$ and $E_{3}=\{u v \mid d(u)=n-1$ and $d(v)=n-1\}$. It is easy to check that $\left|E_{1}\right|=(k(k-1) / 2)-k,\left|E_{2}\right|=(n-k) k$ and $\left|E_{3}\right|=(n-k)(n-k-1)$ /2. Also diam $\left(K d_{n}(k)\right)=2$.

Therefore by the equality part of Theorem 1 ,

$$
\begin{aligned}
H S\left(K d_{n}(k)\right) & =\sum_{u v \in E_{1}} \frac{2}{4 n-4-[d(u)+d(v)]}+\sum_{u v \in E_{2}} \frac{2}{4 n-4-[d(u)+d(v)]}+\sum_{u v \in E_{3}} \frac{2}{4 n-4-[d(u)+d(v)]} \\
& =\frac{2((k(k-1) / 2)-k)}{2 n+2}+\frac{2 k(n-k)}{2 n}+\frac{2((n-k)(n-k-1) / 2)}{2 n-2} \\
& =\frac{k(k-3)}{2 n+2}+\frac{k(n-k)}{n}+\frac{(n-k)(n-k-1)}{2 n-2} .
\end{aligned}
$$

Note that $H S\left(K d_{4}(3)\right)=3 / 4$ and the graphs $K d_{3}(3)$ and $K d_{4}(4)$ are disconnected.

\section{Correlation between harmonic status index and boiling point of paraffins}

The properties of graphs can be used in the study of quantitative structure-property relationship (QSPR) and quantitative structure-activity relationship (QSAR) of the molecules [6]. In this section we study the correlation between the boiling point (BP) of the paraffin hydrocarbons and the harmonic status index of the corresponding molecular graphs.

Table 1. Boiling point and harmonic status index of paraffins

\begin{tabular}{|l|c|c|}
\hline \multicolumn{1}{|c|}{ Paraffin } & $\begin{array}{c}\text { Boiling } \\
\text { point } \\
\text { (BP) }{ }^{\mathbf{0}} \mathbf{C}\end{array}$ & $\begin{array}{c}\text { Harmonic } \\
\text { status index } \\
\text { (HS) }\end{array}$ \\
\hline 3-methylpentane & 62.9 & 0.542 \\
\hline 2,2 -dimethylbutane & 50 & 0.653 \\
\hline 2,3 dimethylbutane & 57.9 & 0.587 \\
\hline 2,2 dimethylpentane & 79 & 0.517 \\
\hline 3,3 dimethylpentane & 86 & 0.549 \\
\hline n-octane & 125 & 0.4226 \\
\hline 3-methylheptane & 118 & 0.406 \\
\hline 3-ethylhexane & 118 & 0.4351 \\
\hline 2,2-dimethylhexane & 107 & 0.44401 \\
\hline 2,4 dimethylhexane & 108 & 0.4386 \\
\hline 2-methyl, 3-ethylpentene & 116 & 0.4535 \\
\hline 2,2,4-trimethylpentene & 99 & 0.4779 \\
\hline n-dodecane & 216.2 & 0.283 \\
\hline
\end{tabular}

Using the data of Table 1, the scatter plot between the boiling point (BP) and harmonic status index (HS) of paraffins is depicted in Figure 4. 


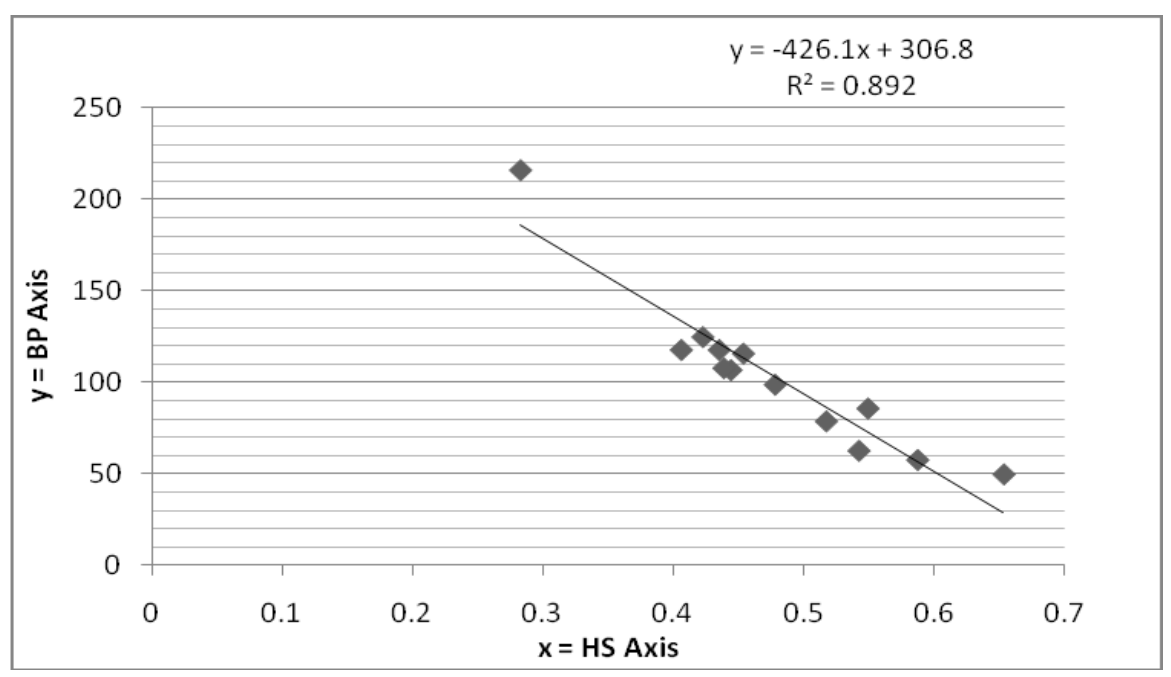

Fig. 4. Regression line between the boiling point (BP) and the harmonic status index (HS) of paraffins

The linear regression between BP and HS is BP $=306.8( \pm 21.742)-426.1( \pm 44.741) \mathrm{HS}$.

The correlation of the boiling point of paraffins with the harmonic status index is good $(\mathrm{R}=0.9444)$.

\section{Acknowledgement}

Authors are grateful to the referee and editor for their useful suggestions. This work was supported by the University Grants Commission (UGC), Govt. of India through research grant under UGCSAP DRS-III for 2016-2021: F.510/3/DRS-III /2016 (SAP-I) Dated: 29th Feb. 2016. The author A. S. Yalnaik is thankful to the University Grants Commission (UGC), Govt. of India for support through Rajiv Gandhi National Fellowship No. F1-17.1/2014-15/RGNF-2014-15-SC-KAR-74909.

\section{References}

[1] J.A. Bondy, U.S.R. Murty, Graph theory with applications. American Elsevier Publishing Co., New York, 1976.

[2] F. Buckley, F. Harary, Distance in Graphs, Addison-Wesley, Redwood, 1990.

[3] R. Chang, Y. Zhu, On the harmonic index and the minimum degree of a graph, Romanian J. Inf. Sci. Tech. 15 (2012) 335-343.

[4] K.C. Das, I. Gutman, Estimating the Wiener index by means of number of vertices of edges and diameter, MATCH Commun. Math. Comput. Chem. 64 (2010) 647-660.

[5] H. Deng et al., On the harmonic index and the chromatic number of a graph, Discrete Appl. Math. 161 (2013) 2740-2744.

[6] J. Devillers, A.T. Balaban (Eds.), Topological Indices and Related Descriptors in QSAR and QSPR, Gordan and Breach, Amsterdam, 1999.

[7] A.A. Dobrynin, R. Entringer, I. Gutman, Wiener index of trees: Theory and Applications, Acta Appl. Math. 66 (2001) 211-249.

[8] S. Fajtlowicz, On conjectures of Graffiti - II, Congr. Numer. 60 (1987) 187-197.

[9] I. Gutman, L. Pavlovic, The energy of some graphs with large number of edges. Bull. Acad. Serbe Sci. Arts. (Cl. Math. Natur.) 118 (1999) 35-50. 
[10] I. Gutman et al., Some recent results in the Theory of the Wiener number, Indian J. Chem. 32A (1993) 651-661.

[11] F. Harary, Status and Contrastatus, Sociometry. 22 (1959) 23-43.

[12] Y. Hu, X. Zhou, On the harmonic index of the unicyclic and bicyclic graphs, Wseas Tran. Math. 12 (2013) 716-726.

[13] G. Indulal, I. Gutman, A. Vijaykumar, On the distance energy of a graph, MATCH Commun. Math. Comput. Chem. 60 (2008) 461-472.

[14] O. Ivanciuc, T.S. Balaban, A.T. Balaban, Design of topological indices, Part 4. Reciprocal distance matrix related local vertex invariants and topological indices, J. Math. Chem. 12 (1993) 309-318.

[15] J. Liu, On the harmonic index of triangle free graphs, Appl. Math. 4 (2013) 1204-1206.

[16] J. Liu, On the harmonic index and diameter of graphs, J. Appl. Math. Phy. 1 (2013) 5-6.

[17] D. Plavsic, S. Nikolic, N. Trinajstic, On the Harary index for the characterization of chemical graphs, J. Math. Chem. 12 (1993) 235-250.

[18] H.S. Ramane, Distance energy of graphs, in: Energies of Graphs - Theory and Applications (Eds.: I. Gutman, X. Li), Univ. Kragujevac, Kragujevac, 2016, pp. 123 - 144.

[19] H.S. Ramane, A.B. Ganagi, H.B. Walikar, Wiener index of graphs in terms of eccentricities, Iranian J. Math. Chem. 4 (2013) 239-248.

[20] H.S. Ramane, V.V. Manjalapur, Note on the bounds on Wiener number of a graph, MATCH Commun. Math. Comput. Chem. 76 (2016) 19-22.

[21] M. Randic, Novel molecular descriptor for structure-property studies, Chem. Phys. Lett. 211 (1993) 478-483.

[22] B. Shwetha Shetty, V. Lokesha, P.S. Ranjini, On the harmonic index of graph operations, Trans. Combin. 4 (2015) 5-14.

[23] H.B. Walikar, V.S. Shigehalli, H.S. Ramane, Bounds on the Wiener number of a graph, MATCH Commun. Math. Comp. Chem. 50 (2004) 117-132.

[24] H. Wiener, Structural determination of paraffin boiling points, J. Amer. Chem. Soc. 69 (1947) 17-20.

[25] L. Zhong, The harmonic index of graphs, Appl. Math. Lett. 25 (2012) 561-566.

[26] Y. Zhu, R. Chang, On the harmonic index of bicyclic conjugated molecular graphs, Filomat. 28(2) (2014) 421-428. 